\title{
Passivity and Passification for Delay Fuzzy System Based on Delay Partitioning Approach
}

\author{
Xiangjie Liu, ${ }^{1}$ Dan Yue, ${ }^{1}$ and Xiuming Yao ${ }^{2}$ \\ ${ }^{1}$ The State Key Laboratory of Alternate Electrical Power System with Renewable Energy Sources, \\ North China Electric Power University, Beijing 102206, China \\ ${ }^{2}$ Department of Automation, North China Electric Power University, Baoding 071003, China
}

Correspondence should be addressed to Xiuming Yao; xiumingyao@gmail.com

Received 17 February 2013; Accepted 28 March 2013

Academic Editor: Constantinos Siettos

Copyright (c) 2013 Xiangjie Liu et al. This is an open access article distributed under the Creative Commons Attribution License, which permits unrestricted use, distribution, and reproduction in any medium, provided the original work is properly cited.

\begin{abstract}
A delay partitioning approach is introduced to solve problems of passivity and passification for continuous T-S fuzzy system with time delay. Our aim is to design a state feedback controller such that the resulting closed system is passive. By constructing a Lyapunov-Krasovskii functional, delay-dependent passivity/passification performance conditions are formulated in terms of linear matrix inequalities (LMIs). Finally, numerical examples are used to illustrate the effectiveness of the proposed approaches which can further reduce conservatism and become more obvious with partitioning getting thinner.
\end{abstract}

\section{Introduction}

The passivity concept was introduced by Willems [1] and developed further by Hill and Moylan [2]. Passivity of nonlinear systems has attracted great interest in the control area mainly because of the link between stability and passivity. The passivity theory provides a nice tool for analyzing the stability of systems and has found applications in diverse areas such as stability, signal processing, chaos control, and synchronization.

Since most physical systems in real world are nonlinear, researchers have been devoting their efforts to the control of nonlinear systems. Among the methods, the fuzzy control has been proven to be effective in dealing with the analysis of nonlinear systems. especially the T-S fuzzy control $[3,4]$. It is denoted by a group of IF-THEN rules that the conventional linear system theory can be applied to the analysis of the class of nonlinear systems, and numerous nonlinear analysis problems have been studied based on this T-S fuzzy model, such as [5-7] reported the problem of stability analysis, and [8-10] investigated the $H_{\infty}$ control designs. References [11-13] mentioned the fault detection of the T-S fuzzy systems. $H_{\infty}$ model reduction is addressed in [14]. The fuzzy controller was carried out via Parallel Distributed Compensation (PDC) technique [15]. Based on the PDC technique, the fuzzy controller can also be designed to guarantee the passivity of T-S fuzzy systems.

On the other hand, the time delay exists naturally in various control systems. Time delays often degrade the system's performance and even cause instability. Therefore, time delays have received great attention in recent years and many researchers have studied various analytical techniques and developed many synthesis methods for timedelay systems. For instance, model reduction is addressed in $[16]$ and filtering problems are investigated in $[17,18]$. So, the passivity and passification analysis of nonlinear systems with time delays is worth to be discussed and researched. To date, researches have gained many results in passivity control of T-S fuzzy systems; the passivity of delayed neural networks is considered in [19], passivity of fuzzy time-delay systems is investigated in [20] which adopt delay moom's inequality, and passive controller design for T-S fuzzy systems is addressed in [21]. The passivity of uncertain fuzzy systems is considered in [22]. However, the above methods still have strong conservation, and it is necessary for us to further study.

In this paper, we adopt a delay partitioning approach to study the passivity and passification of T-S fuzzy systems with time delay. Based on this idea, we can further reduce the 
conservatism, and it becomes even less conservative when the partitioning goes finer. The results of this paper are given in terms of LMIs. The rest of the paper is organized as follows. In Section 2, the problem to be studied is stated and some preliminaries are presented. Passivity analysis results are presented in Section 3. Based on the results obtained in Section 3, we design the controller in Section 4. In Section 5, numerical examples are given to demonstrate the effectiveness of the theoretical results. Finally, conclusions are drawn in Section 6.

Notations. Throughout the paper, $A^{-1}$ and $A^{T}$ denote the inverse and transpose of a square matrix $A . R^{n}$ denotes the $n$-dimensional Euclidean apace and $\|\cdot\|$ refers to the Euclidean vector norm. The notation $A>0$ is used to define a symmetric positive definite matrix and sym $(A)$ is defined as $A+A^{T}$. Matrices are assumed to have compatible dimensions.

\section{Problem Statement and Preliminaries}

Consider the T-S fuzzy system with time delay has the following form.

Plant Rule $i$ :

IF $Z_{1}(t)$ is $M_{i 1}$ and $\ldots$ and $Z_{p}(t)$ is $M_{i p}$ THEN

$$
\begin{gathered}
\dot{x}(t)=A_{i} x(t)+A_{d i} x(t-h)+B_{i} u(t)+B_{1 i} \omega(t), \\
y(t)=C_{i} x(t)+C_{d i} x(t-h)+D_{i} \omega(t), \\
x(t)=\varphi(t), \quad t \in[-h, 0], \\
i=1, \ldots, r,
\end{gathered}
$$

where $x(t) \in R^{n}$ is the state vector; $u(t)$ is the control input vector; $h$ is a time delay; $\varphi(t)$ is the initial condition.

Controller Rule $i$ :

IF $z_{1}(t)$ is $M_{i 1}$ and... and $z_{p}(t)$ is $M_{i p}$ THEN

$$
u(t)=K_{i} x(t), \quad i=1, \ldots, r,
$$

where $M_{i j}$ is the fuzzy set; $r$ is the number of IF-THEN rules; $z(t)=\left[z_{1}(t), z_{2}(t), \ldots, z_{p}(t)\right]$ is the premise variables vector; $K_{i}, i=1, \ldots, r$ are constant matrices representing state-feedback control gains. Let $\lambda_{i}(t)$ be the normalized membership function of the fuzzy set

$$
\lambda_{i}(t)=\frac{\prod_{j=1}^{p} M_{i j}\left(z_{j}(t)\right)}{\sum_{i=1}^{r}\left\{\prod_{j=1}^{p} M_{i j}\left(z_{j}(t)\right)\right\}},
$$

where $M_{i j}\left(z_{j}(t)\right)$ is the grade of membership function of $z_{j}(t)$ in $M_{i j}(t)$. It is assumed that $\prod_{j=1}^{p} M_{i j}\left(z_{j}(t)\right) \geq 0, i=1, \ldots, r$, and $\sum_{i=1}^{r}\left\{\prod_{j=1}^{p} M_{i j}\left(z_{j}(t)\right)\right\}>0$ for all $t$. Therefore, $\lambda_{i}(t) \geq 0$ and $\sum_{i=1}^{r} \lambda_{i}(t)=1$ for all $t$. By applying (3) into (1), the fuzzy system can be expressed as

$$
\begin{gathered}
\dot{x}(t)=A(t) x(t)+A_{d}(t) x(t-h) \\
+B(t) u(t)+B_{1}(t) \omega(t), \\
y(t)=C(t) x(t)+C_{d}(t) x(t-h)+D(t) \omega(t)
\end{gathered}
$$

with

$$
\begin{array}{cc}
A(t)=\sum_{i=1}^{r} \lambda_{i}(t) A_{i}, & A_{d}(t)=\sum_{i=1}^{r} \lambda_{i}(t) A_{d i}, \\
B(t)=\sum_{i=1}^{r} \lambda_{i}(t) B_{i}, & B_{1}(t)=\sum_{i=1}^{r} \lambda_{i}(t) B_{1 i}, \\
C(t)=\sum_{i=1}^{r} \lambda_{i}(t) C_{i}, & C_{d}(t)=\sum_{i=1}^{r} \lambda_{i}(t) C_{d i}, \\
D(t)=\sum_{i=1}^{r} \lambda_{i}(t) D_{i} .
\end{array}
$$

By applying (2) into (4), we can get the following closed-loop system:

$$
\begin{gathered}
\dot{x}(t)=\sum_{i=1}^{r} \sum_{j=1}^{r} \lambda_{i}(z(t)) \lambda_{j}(z(t)) \\
\times\left[\left(A_{i}+B_{i} K_{j}\right) x(t)\right. \\
\left.+A_{d i} x(t-h)+B_{1 i} \omega(t)\right], \\
y(t)=\sum_{i=1}^{r} \lambda_{i}(z(t))\left[C_{i} x(t)+C_{d i} x(t-h)+D_{i} \omega(t)\right] .
\end{gathered}
$$

Before formulating the main problem, we first give the following definition.

Definition 1 (Li et al. [20]). The fuzzy system (1) is called passive if there exists a scalar $\gamma \geq 0$ such that

$$
2 \int_{0}^{T} \omega(s)^{T} y(s) d s \geq-\gamma \int_{0}^{T} \omega^{T}(s) \omega(s) d s
$$

for all $T \geq 0$

\section{Passivity Analysis}

The problems to be addressed in this paper can be expressed as follows.

Problem 1 (passivity analysis). Given the feedback controller gain matrices $K_{i}, i=1, \ldots, r$ in (2), determine under what conditions the closed-loop system (6) is passive for all $T \geq 0$ in the sense of Definition 1.

Problem 2 (passification). Determine the feedback controller gain matrices, $K_{i}, i=1, \ldots, r$ in (2), such that the closed-loop system (6) is passive for all $T \geq 0$ in the sense of Definition 1 .

In this section, we will present a sufficient condition in terms of LMIs, under which the closed-loop system (6) is passive. 
Theorem 2. Given matrices $K_{i}$, an integer $m \geq 1$ and a scalar $h>0$, if there exist symmetric positive definite matrices $P, Q_{i}$, $Z_{i}, R_{i}$ and matrices $S_{1 i}, S_{2 i}$ and scalar $\gamma>0$, satisfying

$$
\begin{aligned}
& {\left[\begin{array}{cc}
\Theta_{i i l k}+\sigma W_{\sigma}^{T} W_{\sigma} & S_{1 i} \\
* & -\frac{m}{h} Z_{i}
\end{array}\right]<0, \quad i, l, k=1, \ldots, r} \\
& {\left[\begin{array}{cc}
\Theta_{i j l k}+\sigma W_{\sigma}^{T} W_{\sigma} & S_{1 i} \\
* & -\frac{m}{h} Z_{i}
\end{array}\right]} \\
& +\left[\begin{array}{cc}
\Theta_{j i l k}+\sigma W_{\sigma}^{T} W_{\sigma} & S_{1 j} \\
* & -\frac{m}{h} Z_{j}
\end{array}\right]<0 \\
& 1 \leq r<j \leq r, \quad l, k=1, \ldots, r, \\
& Z_{i}<R_{j}, \quad i, j=1, \ldots, r,
\end{aligned}
$$

where

$$
\begin{aligned}
& \Omega_{i j l k}=W p^{T} \stackrel{\wedge}{P} W p+\frac{h}{m} W r^{T} R_{i} W r+W q_{1}^{T} Q_{i} W q_{1} \\
& -W q_{2}^{T} Q_{l} W q_{2}+\operatorname{sym}\left(s_{i} W s_{j k}\right), \\
& \Theta_{i j l k}=\Omega_{i j l k}-\left(W_{1} C_{j}^{T} W_{2}+W_{2}^{T} C_{j} W_{1}^{T}\right. \\
& +W_{2}^{T} D_{j}^{T} W_{2}+W_{2}^{T} D_{j} W_{2} \\
& \left.+W_{2}^{T} C_{d j} W_{3}+W_{3}^{T} C_{d j}^{T} W_{2}+\gamma W_{2}^{T} W_{2}\right), \\
& W p=\left(\begin{array}{cccc}
I_{n} & O_{n, m n} & O_{n, n} & O_{n, n} \\
O_{n, n} & O_{n, m n} & I_{n} & O_{n, n} \\
O_{n, n} & O_{n, m n} & O_{n, n} & O_{n, n}
\end{array}\right), \\
& \hat{P}=\left(\begin{array}{ccc}
O_{n, n} & P & O_{n, n} \\
P & O_{n, n} & O_{n, n} \\
O_{n, n} & O_{n, n} & O_{n, n}
\end{array}\right), \quad W_{\sigma}=\left(I_{n}, O_{n, m n+2 n}\right), \\
& W r=\left(O_{n, m n+n}, I_{n}, O_{n, n}\right), \quad W q_{1}=\left(I_{m n}, O_{m n, 3 n}\right) \text {, } \\
& W q_{2}=\left(O_{m n, n}, I_{m n}, O_{m n, 2 n}\right), \quad S_{i}=\left[\begin{array}{ll}
S_{1 i} & S_{2 i}
\end{array}\right],
\end{aligned}
$$

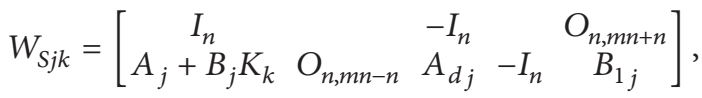

$$
\begin{aligned}
& W_{1}=\left(\begin{array}{c}
I_{n} \\
O_{m n+2 n, n}
\end{array}\right), \quad W_{2}=\left(O_{n, m n+2 n}, I_{n}\right) \\
& W_{3}=\left(O_{n, m n}, I_{n}, O_{n, 2 n}\right) \text {. }
\end{aligned}
$$

Then the fuzzy system (1) is passive in the sense of Definition 1 for the time delay $0 \leq \tau \leq h$.
Proof. Choose a Lyapunov-Krasovskii functional as $V(t)=$ $V_{1}(t)+V_{2}(t)+V_{3}(t)$ with

$$
\begin{gathered}
V_{1}(t)=x^{T}(t) P x(t), \\
V_{2}(t)=\int_{-h / m}^{0} \int_{t+\beta}^{t} \dot{x}^{T}(\alpha) R(\alpha) \dot{x}(\alpha) d \alpha d \beta, \\
V_{3}(t)=\int_{t-h / m}^{t} \gamma^{T}(\alpha) Q(\alpha) \gamma(\alpha) d \alpha,
\end{gathered}
$$

where

$$
\gamma(t)=\left[\begin{array}{llll}
x^{T}(t) & x^{T}\left(t-\frac{h}{m}\right) & \cdots & x^{T}\left(t-\frac{m-1}{m} h\right)
\end{array}\right]^{T} .
$$

The time-derivative of $V(t)$ along the trajectory of the system in (6) is given by

$$
\begin{gathered}
\dot{V}_{1}=\dot{x}^{T}(t) P x(t)+x^{T}(t) P \dot{x}(t), \\
\dot{V}_{2}=\frac{h}{m} \dot{x}^{T}(t) R(t) \dot{x}(t)-\int_{t-h / m}^{t} \dot{x}^{T}(\alpha) R(\alpha) \dot{x}(\alpha) d \alpha \\
\dot{V}_{3}=\gamma^{T}(t) Q(t) \gamma(t)-\gamma^{T}\left(t-\frac{h}{m}\right) Q\left(t-\frac{h}{m}\right) \gamma\left(t-\frac{h}{m}\right) .
\end{gathered}
$$

Define

$$
\begin{gathered}
\xi(t)=\left[\begin{array}{llll}
\gamma^{T}(t) & x^{T}(t-h) & \dot{x}^{T}(t) & \omega^{T}(t)
\end{array}\right]^{T}, \\
\omega(t)=\left[\begin{array}{lll}
\omega_{1}(t) & \cdots & \omega_{n}(t)
\end{array}\right]^{T}
\end{gathered}
$$

and according to the Newton-Leibniz formula and the system in (6), we have

$$
\begin{aligned}
& \Pi_{1}=2 \xi^{T}(t) S_{1}(t) \\
& \times\left[x(t)-x\left(t-\frac{h}{m}\right)-\int_{t-h / m}^{t} \dot{x}(\alpha) d \alpha\right]=0, \\
& \Pi_{2}=2 \xi^{T}(t) S_{2}(t)[(A(t)+B(t) K(t)) x(t) \\
& +A_{d}(t) x(t-h) \\
& \left.+B_{1}(t) \omega(t)-\dot{x}(t)\right]=0,
\end{aligned}
$$

$$
\begin{aligned}
\Pi_{3}= & \frac{h}{m} \xi^{T}(t) S_{1}(t) Z^{-1}(t) S_{1}^{T}(t) \xi(t) \\
& \quad-\int_{t-h / m}^{t} \xi^{T}(t) S_{1}(t) Z^{-1}(t) S_{1}^{T}(t) \xi(t) d \alpha=0 .
\end{aligned}
$$


Therefore

$$
\begin{aligned}
\dot{V}(t) & \leq \dot{V}_{1}(t)+\dot{V}_{2}(t)+\dot{V}_{3}(t)+\Pi_{1}+\Pi_{2}+\Pi_{3} \\
= & \dot{x}^{T}(t) P x(t)+x^{T}(t) P \dot{x}(t)+\frac{h}{m} \dot{x}^{T}(t) R(t) \dot{x}(t) \\
& -\int_{t-h / m}^{t} \dot{x}^{T}(\alpha) R(\alpha) \dot{x}(\alpha) d \alpha+\gamma^{T}(t) Q(t) \gamma(t) \\
& -\gamma^{T}\left(t-\frac{h}{m}\right) Q\left(t-\frac{h}{m}\right) \gamma\left(t-\frac{h}{m}\right)+2 \xi^{T}(t) S_{1}(t) \\
& \times\left[x(t)-x\left(t-\frac{h}{m}\right)-\int_{t-h / m}^{t} \dot{x}(\alpha) d \alpha\right] \\
& +2 \xi^{T}(t) S_{2}(t) \\
& \times\left[(A(t)+B(t) K(t)) x(t)+A_{d}(t) x(t-h)\right. \\
& -\int_{t-h / m}^{t} \xi^{T}(t) S_{1}(t) Z^{-1}(t) S_{1}^{T}(t) \xi(t) d \alpha \\
& \left.+\int_{1}^{t}(t) \omega(t)-\dot{x}(t)\right] \\
& +\frac{h}{m} \xi^{T}(t) S_{1}(t) Z^{-1}(t) S_{1}^{T}(t) \xi(t) \\
& -\int_{t-h / m}^{t} \xi^{T}(t) S_{1}(t) Z^{-1}(t) S_{1}^{T}(t) \xi(t) d \alpha \\
& \Lambda(t)+\frac{h}{m} \xi^{T}(t) S_{1}(t) Z^{-1}(t) S_{1}^{T}(t) \xi(t) \\
& +\alpha(\alpha) d \alpha \\
& \\
& \\
& \\
&
\end{aligned}
$$

If

$$
Z(t)<R(\alpha)
$$

Then

$$
\begin{gathered}
Z^{-1}(t)>R^{-1}(\alpha), \\
-\int_{t-h / m}^{t} \xi^{T}(t) S_{1}(t) Z^{-1}(t) S_{1}^{T}(t) \xi(t) d \alpha \\
<-\int_{t-h / m}^{t} \xi^{T}(t) S_{1}(t) R^{-1}(\alpha) S_{1}^{T}(t) \xi(t) d \alpha .
\end{gathered}
$$

So, we can obtain

$$
\begin{aligned}
\dot{V}(t) \leq \Lambda & (t)+\frac{h}{m} \xi^{T}(t) S_{1}(t) Z^{-1}(t) S_{1}^{T}(t) \xi(t) \\
-\int_{t-h / m}^{t} & \left(\dot{x}^{T}(\alpha) R(\alpha)+\xi^{T}(t) S_{1}(t)\right) \times R^{-1}(\alpha) \\
& \times\left(R(\alpha) \dot{x}(\alpha)+S_{1}^{T}(t) \xi(t)\right) d \alpha,
\end{aligned}
$$

where

$$
\begin{aligned}
\Lambda(t)= & \dot{x}^{T}(t) P x(t)+x^{T}(t) P \dot{x}(t)+\frac{h}{m} \dot{x}^{T}(t) R(t) \dot{x}(t) \\
& +\gamma^{T}(t) Q(t) \gamma(t)-\gamma^{T}\left(t-\frac{h}{m}\right) Q\left(t-\frac{h}{m}\right) \\
& \times \gamma\left(t-\frac{h}{m}\right)+2 \xi^{T}(t) S_{1}(t)\left[x(t)-x\left(t-\frac{h}{m}\right)\right] \\
& +2 \xi^{T}(t) S_{2}(t) \\
& \times[(A(t)+B(t) K(t)) x(t) \\
& \left.\quad+A_{d}(t) x(t-h)+B_{1}(t) \omega(t)-\dot{x}(t)\right] .
\end{aligned}
$$

Besides

$$
\begin{gathered}
\dot{x}(t)=\left(O_{n, m n+n}, I_{n}, O_{n, n}\right) \xi(t), \\
x(t)=\left(I_{n}, O_{n, m n+2 n}\right) \xi(t), \\
\gamma(t)=\left(I_{m n}, O_{m n, 3 n}\right) \xi(t), \\
\gamma\left(t-\frac{h}{m}\right)=\left(O_{m n, n}, I_{m n}, O_{m n, 2 n}\right) \xi(t) .
\end{gathered}
$$

Then

$$
\Lambda(t)=\xi^{T}(t) \Omega(t) \xi(t),
$$

$\Omega(t)=W_{p}^{T} \stackrel{\wedge}{P} W_{p}+\frac{h}{m} W_{r}^{T} R(t) W_{r}+W_{q 1}^{T} Q(t) W_{q 1}$ $-W_{q 2}^{T} Q\left(t-\frac{h}{m}\right) W_{q 2}+\operatorname{sym}\left(S(t) W_{S}(t)\right)$, $\widehat{P}=\left(\begin{array}{ccc}O_{n, n} & P & O_{n, n} \\ P & O_{n, n} & O_{n, n} \\ O_{n, n} & O_{n, n} & O_{n, n}\end{array}\right), \quad S(t)=\left[\begin{array}{ll}S_{1}(t) & S_{2}(t)\end{array}\right]$

$$
W p=\left(\begin{array}{cccc}
I_{n} & O_{n, m n} & O_{n, n} & O_{n, n} \\
O_{n, n} & O_{n, m n} & I_{n} & O_{n, n} \\
O_{n, n} & O_{n, m n} & O_{n, n} & O_{n . n}
\end{array}\right)
$$

$W r=\left(O_{n, m n+n}, I_{n}, O_{n, n}\right), \quad W q_{1}=\left(I_{m n}, O_{m n, 3 n}\right)$,

$$
W q_{2}=\left(O_{m n, n}, I_{m n}, O_{m n, 2 n}\right) \text {, }
$$

$W_{S}(t)=\left[\begin{array}{ccccc}I_{n} & & -I_{n} & O_{n, m n+n} \\ A(t)+B(t) K(t) & O_{n, m n-n} & A_{d}(t) & -I_{n} & B_{1}(t)\end{array}\right]$. 
Besides

$$
\begin{aligned}
2 \omega^{T}(t) & y(t)+\gamma \omega^{T}(t) \omega(t) \\
= & x^{T}(t) C_{i}^{T} \omega(t)+\omega^{T}(t) C_{i} x(t) \\
& +\omega^{T}(t)\left(D_{i}^{T}+D_{i}\right) \omega(t)+\omega^{T}(t) C_{d i} x(t-h) \\
& +x^{T}(t-h) C_{d i}^{T} \omega(t)+\gamma \omega^{T}(t) \omega(t) \\
= & \xi^{T}(t)\left[W_{1} C_{i}^{T} W_{2}+W_{2}^{T} C_{i} W_{1}^{T}+W_{2}^{T}\left(D_{i}^{T}+D_{i}\right) W_{2}\right. \\
& \left.\quad+W_{2}^{T} C_{d i} W_{3}+W_{3}^{T} C_{d i}^{T} W_{2}+\gamma W_{2}^{T} W_{2}\right] \xi(t),
\end{aligned}
$$

where

$$
\begin{gathered}
W_{1}=\left(\begin{array}{c}
I_{n} \\
O_{m n+2 n, n}
\end{array}\right), \quad W_{2}=\left(O_{n, m n+2 n}, I_{n}\right), \\
W_{3}=\left(O_{n, m n}, I_{n}, O_{n, 2 n}\right) .
\end{gathered}
$$

So, we have

$$
\begin{gathered}
\dot{V}(t)-2 \omega^{T}(t) y(t)-\lambda \omega^{T}(t) \omega(t) \\
\leq \xi^{T}(t) \Theta(t) \xi(t)+\frac{h}{m} \xi^{T}(t) S_{1}(t) Z^{-1}(t) S_{1}^{T}(t) \xi(t) \\
-\int_{t-h / m}^{t}\left(\dot{x}^{T}(\alpha) R(\alpha)+\xi^{T}(t) S_{1}(t)\right) \times R^{-1}(\alpha) \\
\times\left(R(\alpha) \dot{x}(\alpha)+S_{1}^{T}(t) \xi(t)\right) d \alpha
\end{gathered}
$$

with

$$
\begin{aligned}
\Theta(t)=\Omega(t)-( & W_{1} C_{i}^{T} W_{2}+W_{2}^{T} C_{i} W_{1}^{T} \\
& +W_{2}^{T}\left(D_{i}+D_{i}^{T}\right) W_{2} \\
& \left.+W_{2}^{T} C_{d i} W_{3}+W_{3}^{T} C_{d i}^{T} W_{2}+\gamma W_{2}^{T} W_{2}\right) .
\end{aligned}
$$

If

$$
\Theta(t)+\sigma W_{\sigma}^{T} W_{\sigma}+\frac{h}{m} S_{1}(t) Z^{-1}(t) S_{1}^{T}(t)<0
$$

then

$$
\begin{aligned}
\Theta(t) & +\frac{h}{m} S_{1}(t) Z^{-1}(t) S_{1}^{T}(t)<-\sigma W_{\sigma}^{T} W_{\sigma} \\
\dot{V}(t) & -2 \omega^{T}(t) y(t)-\gamma \omega^{T}(t) \omega(t) \\
& <\xi^{T}(t) \Theta(t) \xi(t)+\frac{h}{m} \xi^{T}(t) S_{1}(t) Z^{-1}(t) S_{1}^{T}(t) \xi(t) \\
& <-\xi^{T}(t) \sigma W_{\sigma}^{T} W_{\sigma} \xi(t)<-\sigma\|x(t)\|^{2}<0 .
\end{aligned}
$$

We can obtain

$$
\dot{V}(t)<2 y^{T}(t) \omega(t)+\gamma \omega^{T}(t) \omega(t) .
$$

It follows by integrating (31) with respect to $t$ over the time period $0 \sim T$ that (7) holds, and hence the delayed fuzzy system (1) is passive in the sense of Definition 1. Our next objective is to convert the inequalities in (18) and (29) to some finite LMIs, then (29) can be rewritten as

$$
\begin{aligned}
& \eta_{i j l k}= \eta_{i i l k}+\left(\eta_{i j l k(i<j)}+\eta_{j i l k(i<j)}\right)<0, \\
& \eta_{i j l k}=\sum_{i=1}^{r} \lambda_{i}(\theta(t)) \sum_{j=1}^{r} \lambda_{j}(\theta(t)) \\
& \times \sum_{l=1}^{r} \lambda_{l}\left(\theta\left(t-\frac{h}{m}\right)\right) \sum_{k=1}^{r} \lambda_{k}(\theta(t)) \\
& \times\left[\Omega_{i j l k}-\left(W_{1} C_{j}^{T} W_{2}+W_{2}^{T} C_{j} W_{1}^{T}\right.\right. \\
&+W_{2}^{T}\left(D_{j}+D_{j}^{T}\right) W_{2} \\
&\left.+W_{2}^{T} C_{d j} W_{3}+W_{3}^{T} C_{d j}^{T} W_{2}+\gamma W_{2}^{T} W_{2}\right) \\
&+\sigma W_{\sigma}^{T} W_{\sigma} \\
&\left.+\frac{h}{m} S_{1 i} Z_{i}^{-1} S_{1 i}^{T}\right] .
\end{aligned}
$$

Equation (18) can be rewritten as

$$
Z_{i}<R_{j}
$$

From the Schur complement, we can get the inequalities (8), (9), and (10), and the proof is completed.

\section{Controller Design}

In this section, fuzzy state feedback controllers will be designed based on the result developed in the previous section.

Theorem 3. Given an integer $m>1$ and scalars $h>0, \tau_{1}$, $\tau_{2}, \ldots, \tau_{(m+3)}$, there exists a fuzzy state feedback controller such that the closed-loop system (4) is passive if there exist symmetric 
positive definite matrices $\bar{P}, \overline{Q_{i}}, \overline{Z_{i}}, \overline{R_{i}}$, and matrices $X, \overline{S_{1 i}}, M_{i}$ and scalars $\gamma>0, \sigma>0$, satisfying

$$
\left[\begin{array}{ccccc}
\varphi_{i i l} & \overline{S_{1 i}} & W_{\sigma}^{T} X^{T} & W_{2}^{T} X^{T} & W_{2}^{T} X^{T} \\
* & -\frac{m}{h} \overline{Z_{i}} & 0 & 0 & 0 \\
* & * & -\sigma^{-1} I_{n} & 0 & 0 \\
* & * & * & D_{i}^{-T}+D_{i}^{-1} & 0 \\
* & * & * & * & \gamma^{-1} I_{n}
\end{array}\right]<0
$$$$
i, l=1, \ldots, r,
$$$$
\left[\begin{array}{ccccc}
\phi_{i j l} & \overline{S_{1 i}} & W_{\sigma}^{T} X^{T} & W_{2}^{T} X^{T} & W_{2}^{T} X^{T} \\
* & -\frac{m}{h} \overline{Z_{i}} & 0 & 0 & 0 \\
* & * & -\sigma^{-1} I_{n} & 0 & 0 \\
* & * & * & D_{i}^{-T}+D_{i}^{-1} & 0 \\
* & * & * & * & \gamma^{-1} I_{n}
\end{array}\right]
$$$$
+\left[\begin{array}{ccccc}
\phi_{j i l} & \overline{S_{1 j}} & W_{\sigma}^{T} X^{T} & W_{2}^{T} X^{T} & W_{2}^{T} X^{T} \\
* & -\frac{m}{h} \overline{Z_{j}} & 0 & 0 & 0 \\
* & * & -\sigma^{-1} I_{n} & 0 & 0 \\
* & * & * & D_{j}^{-T}+D_{j}^{-1} & 0 \\
* & * & * & * & \gamma^{-1} I_{n}
\end{array}\right]<0
$$$$
1 \leq i<j \leq r, \quad l=1, \ldots, r,
$$

$$
\overline{Z_{i}}<\overline{R_{j}}, \quad i, j=1, \ldots, r,
$$

where

$$
\begin{aligned}
& \varphi_{i j l}=W_{p}^{T} \stackrel{\wedge}{P} W_{p}+\frac{h}{m} W_{r}^{T} \overline{R_{i}} W_{r}+W_{q 1}^{T} \overline{Q_{i}} W_{q 1}-W_{q 2}^{T} \overline{Q_{l}} W_{q 2} \\
& +\operatorname{sym}\left(V_{i} \overline{W_{S i j}}-W_{1} X^{T} C_{i}^{T} W_{2}-W_{2}^{T} C_{d i} X W_{3}\right), \\
& \widehat{P}=\left(\begin{array}{ccc}
O_{n, n} & \bar{P} & O_{n, n} \\
\bar{P} & O_{n, n} & O_{n, n} \\
O_{n, n} & O_{n, n} & O_{n, n}
\end{array}\right), \quad V_{i}=\left[\begin{array}{ll}
\overline{S_{1 i}} & U
\end{array}\right], \\
& U=\left[\begin{array}{llll}
\tau_{1} I_{n} & \tau_{2} I_{n} & \cdots & \tau_{(m+3)} I_{n}
\end{array}\right]^{T}, \\
& \overline{W_{S i j}}=\left[\begin{array}{ccccc}
I_{n} & -I_{n} & O_{n, m n+n} & & \\
\hline A_{i} X+B_{i} M_{j} & O_{n, m n-n} & A_{d i} X & -X & B_{1 i}
\end{array}\right] \text {. }
\end{aligned}
$$

If the above conditions are feasible, the gains of the controller are given by

$$
K_{i}=M_{i} X^{-1}, \quad i=1, \ldots, r .
$$

Proof. Assume that $X$ is invertible, define $S=X^{-T}$, and

$$
\begin{gathered}
G 1=\operatorname{diag}\left\{S, \ldots, S, I_{n}, S, S, S, S\right\} \in R^{(m n+7 n) \times(m n+7 n)}, \\
G 2=\operatorname{diag}\left\{S, \ldots, S, I_{n}\right\} \in R^{(m n+3 n) \times(m n+3 n)}, \\
G 3=\operatorname{diag}\{S, \ldots, S\} \in R^{m n \times m n} .
\end{gathered}
$$

Premultiplying and postmultiplying (34) with $G_{1}$ and $G_{1}^{T}$, then we obtain

$$
\left[\begin{array}{ccccc}
\Delta & G_{2} \overline{S_{1 i}} S^{T} & G_{2} W_{\sigma}^{T} X^{T} S^{T} & G_{2} W_{2}^{T} X^{T} S^{T} & G_{2} W_{2}^{T} X^{T} S^{T} \\
* & -\frac{m}{h} S \overline{Z_{i}} S^{T} & 0 & 0 & 0 \\
* & * & -\sigma^{-1} S S^{T} & 0 & 0 \\
* & * & * & S\left(D_{i}^{-T}+D_{i}^{-1}\right) S^{T} & 0 \\
* & * & * & * & \gamma^{-1} S S^{T}
\end{array}\right]<0 \text {, }
$$

where

$$
\begin{gathered}
\Delta=G_{2} \phi_{i i l} G_{2}^{T}, \\
G_{2} \phi_{i i l} G_{2}^{T}=W_{p}^{T}\left[\begin{array}{ccc}
O_{n, n} & S \bar{P} S^{T} & O_{n, n} \\
S \bar{P} S^{T} & O_{n, n} & O_{n, n} \\
O_{n, n} & O_{n, n} & O_{n, n}
\end{array}\right] W_{p} \\
+\frac{h}{m} W_{r}^{T} S \overline{R_{i}} S^{T} W_{r} \\
+W_{q 1}^{T} G_{3} \overline{Q_{i}} G_{3}^{T} W_{q 1}-W_{q 2}^{T} G_{3} \overline{Q_{l}} G_{3}^{T} W_{q 2} \\
+\operatorname{sym}\left(G_{2} V_{i} \overline{W_{S i i}} G_{2}^{T}\right)-W_{1} C_{i}^{T} W_{2} \\
-W_{2}^{T} C_{i} W_{1}^{T}-W_{3}^{T} C_{d i}^{T} W_{2}-W_{2}^{T} C_{d i} W_{3}, \\
G_{2} W_{\sigma}^{T} X^{T} S^{T}=W_{\sigma}^{T} S^{T}, \quad G_{2} W_{2}^{T} X^{T} S^{T}=W_{2}^{T} S^{T} .
\end{gathered}
$$

We defining

$$
\begin{gathered}
P=S \bar{P} S^{T}, \quad R_{i}=S \overline{R_{i}} S^{T}, \quad Q_{i}=G_{3} \overline{Q_{i}} G_{3}^{T}, \\
S_{1 i}=G_{2} \overline{S_{1 i}} S^{T}, \quad Z_{i}=S \overline{Z_{i}} S^{T}, \\
S_{2 i}=\left[\begin{array}{lllll}
\tau_{1} S^{T} & \tau_{2} S^{T} & \cdots & \tau_{(m+2)} S^{T} & \tau_{(m+3)} I_{n}
\end{array}\right]^{T} .
\end{gathered}
$$


Then

$$
\begin{aligned}
& G_{2} V_{i} W_{S i i} G_{2}^{T} \\
&=G_{2} \overline{S_{1 i}}\left[\begin{array}{lllll}
I_{n} & -I_{n} & O_{n, m n+n}
\end{array}\right] G_{2}^{T} \\
&+G_{2} U\left[\begin{array}{lllll}
A_{i} X+B_{i} M_{i} & O_{n, m n-n} & A_{d i} X & -X & B_{1 i}
\end{array}\right] G_{2}^{T} \\
&= G_{2} \overline{S_{1 i}} S^{T}\left[\begin{array}{lllll}
I_{n} & -I_{n} & O_{n, m n+n}
\end{array}\right] \\
&+S_{2 i}\left[\begin{array}{lllll}
A_{i}+B_{i} K_{k} & O_{n, m n-n} & A_{d i} & -I_{n} & B_{1 i}
\end{array}\right] \\
&= {\left[\begin{array}{ll}
S_{1 i} & S_{2 i}
\end{array}\right]\left[\begin{array}{ccccc}
\frac{I_{n}}{A_{i}+B_{i} K_{k}} & O_{n, m n-n} & A_{d i} & -I_{n} & B_{1 i}
\end{array}\right] }
\end{aligned}
$$

then

$$
\begin{aligned}
G_{2} \varphi_{i i l} G_{2}^{T}= & W_{p}^{T}\left[\begin{array}{ccc}
O_{n, n} & P & O_{n, n} \\
P & O_{n, n} & O_{n, n} \\
O_{n, n} & O_{n, n} & O_{n, n}
\end{array}\right] W_{p}+\frac{h}{m} W_{r}^{T} R_{i} W_{r} \\
& +W_{q 1}^{T} Q_{i} W_{q 1}-W_{q 2}^{T} Q_{l} W_{q 2}+\operatorname{sym}\left(G_{2} V_{i} \overline{W_{S i i}} G_{2}^{T}\right) \\
& -W_{1} C_{i}^{T} W_{2}-W_{2}^{T} C_{i} W_{1}^{T} \\
& -W_{3}^{T} C_{d i}^{T} W_{2}-W_{2}^{T} C_{d i} W_{3} .
\end{aligned}
$$

Thus, by the Schur complement, we can obtain (40) is equivalent to (8). Pre- and postmultiplying (35) with $G_{1}$ and $G_{1}^{T}$, we obtain (9), pre- and postmultiplying (36) with $S$ and $S^{T}$, we obtain (10). The proof is completed.

\section{Numerical Example}

Without delay and uncertainty, Example 1 designs different passive controllers by applying the theorem of our paper and the literature [22], respectively. We can compare the region of feasible solution.

Example 1. Consider a fuzzy system of the following form.

Plant Rules:

Rule 1: IF $x_{1}(t)$ is $M_{1}$, THEN

$$
\begin{gathered}
\dot{x}(t)=A_{1} x(t)+B_{1} u(t)+B_{11} \omega(t), \\
y(t)=C_{1} x(t)+D_{1} \omega(t) .
\end{gathered}
$$

Rule 2: IF $x_{1}(t)$ is $M_{2}$, THEN

$$
\begin{gathered}
\dot{x}(t)=A_{2} x(t)+B_{2} u(t)+B_{12} \omega(t), \\
y(t)=C_{2} x(t)+D_{2} \omega(t)
\end{gathered}
$$

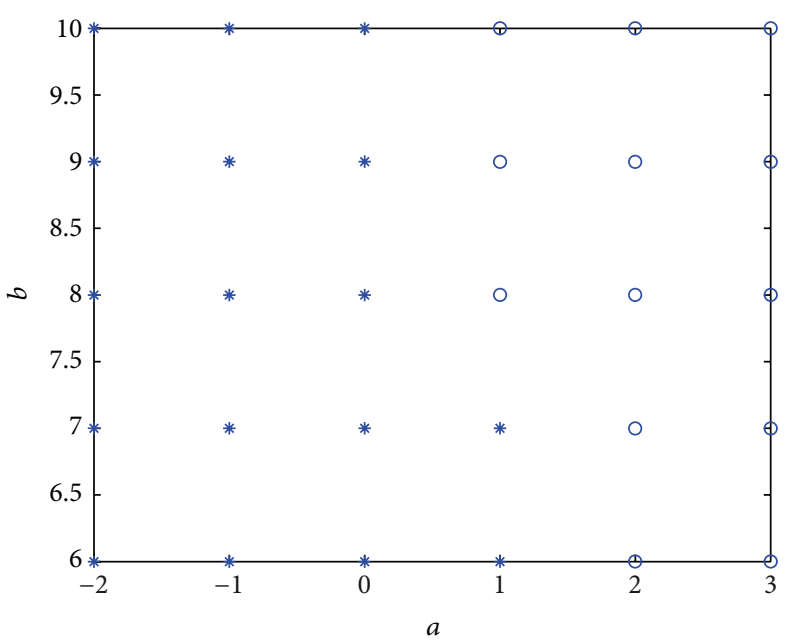

Figure 1: The feasible region based on Theorem 2.

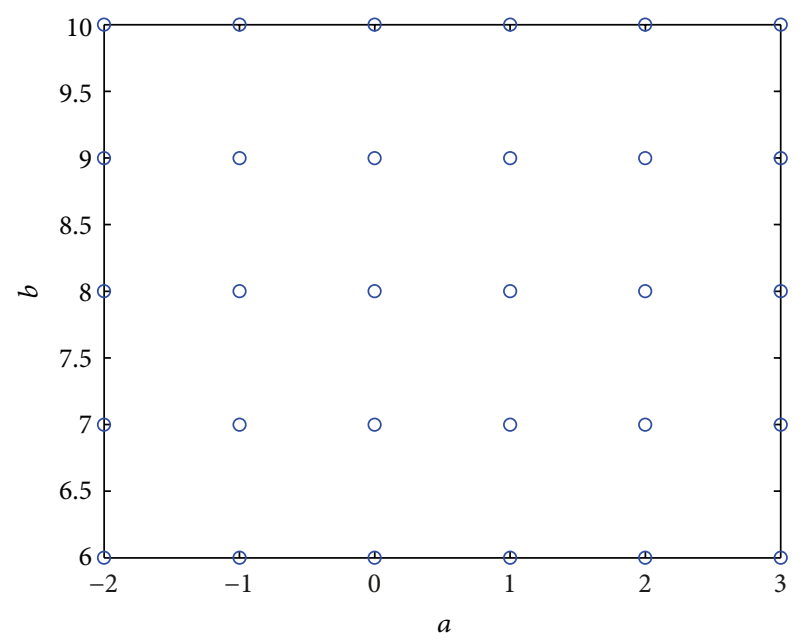

FIgURE 2: The feasible region based on [22].

with

$$
\begin{aligned}
& A_{1}=\left[\begin{array}{ccc}
a & -0.02 & 1 \\
1 & 0 & 5 \\
1 & 2 & 3
\end{array}\right], \quad A_{2}=\left[\begin{array}{ccc}
-0.225 & -0.02 & 0 \\
1 & 0 & 3 \\
1 & 1 & 1
\end{array}\right], \\
& B_{1}=\left[\begin{array}{l}
1 \\
0 \\
b
\end{array}\right], \quad B_{2}=\left[\begin{array}{l}
1 \\
0 \\
2
\end{array}\right], \quad B_{11}=\left[\begin{array}{lll}
1 & 0 & 0 \\
1 & 2 & 0 \\
0 & 1 & 0
\end{array}\right], \\
& B_{12}=\left[\begin{array}{lll}
1 & 0 & 0 \\
1 & 2 & 3 \\
0 & 1 & 2
\end{array}\right], \quad C_{1}=C_{2}=\left[\begin{array}{ccc}
2.5 & 0 & 0 \\
0 & 2.5 & 0 \\
1 & 2 & 1
\end{array}\right], \\
& D_{1}=D_{2}=\left[\begin{array}{lll}
2 & 0 & 0 \\
0 & 2 & 0 \\
1 & 1 & 1
\end{array}\right], \quad a \in[-2,3], b \in[6,10] .
\end{aligned}
$$

We can obtain Figure 1 by applying our method and obtain Figure 2 by applying method in [22]. Symbol "*” shows that 
feasible solution exists at that point; symbol "o" shows that feasible solution does not exist at that point.

It is obvious that Theorem 3 can relax the conditions in [22], and our method futher reduced the conservatism.

Example 2 (Li et al. [20]). Consider a delayed fuzzy system of the following form.

Plant Rules:

Rule 1: IF $x_{1}(t)$ is $M_{1}$, THEN

$$
\begin{gathered}
\dot{x}(t)=A_{1} x(t)+A_{d 1} x(t-h)+B_{1} u(t)+B_{11} \omega(t), \\
y(t)=C_{1} x(t)+C_{d 1} x(t-h)+D_{1} \omega(t) .
\end{gathered}
$$

Rule 2: IF $x_{1}(t)$ is $M_{2}$, THEN

$$
\begin{gathered}
\dot{x}(t)=A_{2} x(t)+A_{d 2} x(t-h)+B_{2} u(t)+B_{12} \omega(t), \\
y(t)=C_{2} x(t)+C_{d 2} x(t-h)+D_{2} \omega(t)
\end{gathered}
$$

with

$$
\begin{gathered}
A_{1}=\left[\begin{array}{cc}
-1 & 0.2 \\
0 & -0.1
\end{array}\right], \quad A_{2}=\left[\begin{array}{cc}
-1 & 0 \\
0.1 & -0.5
\end{array}\right], \\
A_{d 1}=\left[\begin{array}{cc}
0.1 & 0.6 \\
0.2 & -0.1
\end{array}\right], \quad A_{d 2}=\left[\begin{array}{cc}
-0.2 & 0.1 \\
0.1 & -0.2
\end{array}\right], \\
B_{1}=\left[\begin{array}{c}
0.2 \\
-0.5
\end{array}\right], \quad B_{2}=\left[\begin{array}{l}
0.3 \\
0.3
\end{array}\right], \quad B_{11}=\left[\begin{array}{cc}
1 & 0 \\
0.2 & 0
\end{array}\right], \\
B_{12}=\left[\begin{array}{cc}
-0.2 & 0 \\
0 & 0
\end{array}\right], \quad C_{1}=\left[\begin{array}{cc}
1 & 0.2 \\
0 & 0
\end{array}\right], \\
C_{2}=\left[\begin{array}{cc}
0.1 & 1 \\
0 & 0
\end{array}\right], \quad C_{d 1}=\left[\begin{array}{cc}
-0.1 & 0.2 \\
0 & 0
\end{array}\right], \\
C_{d 2}=\left[\begin{array}{cc}
0.1 & -0.1 \\
0 & 0
\end{array}\right], \quad D_{1}=\left[\begin{array}{cc}
0.5 & 0 \\
0 & 0
\end{array}\right],
\end{gathered}
$$

we let $h=7.5, m=2, \tau_{1}=10, \tau_{2}=0, \tau_{3}=5, \tau_{4}=10, \tau_{5}=10$ and we can obtain

$$
\begin{gathered}
P=\left[\begin{array}{cc}
29.0804 & 1.8737 \\
1.8737 & 3.7272
\end{array}\right], \quad X=\left[\begin{array}{ll}
1.8020 & 0.0328 \\
0.1385 & 0.4057
\end{array}\right], \\
\gamma=237.5809 .
\end{gathered}
$$

By using the delay-dependent criterion Theorem 3 of [20], we can obtain $\gamma=604.9523$ when $\tau=7.5$. And applying our result, $\gamma=237.5809$ is obtained. We can see that our results is not conservative since $\gamma$ is much smaller than the result of [20].

Example 3. Consider a delayed fuzzy system of the following form.

Plant Rules:

Rule 1: IF $x_{2}(t)$ is $M_{1}$, THEN

$$
\begin{gathered}
\dot{x}(t)=A_{1} x(t)+A_{d 1} x(t-h)+B_{1} u(t)+B_{11} \omega(t), \\
y(t)=C_{1} x(t)+D_{1} \omega(t) .
\end{gathered}
$$

TABLE 1: Allowable maximum time delay $h$.

\begin{tabular}{lc}
\hline Methods & The upper bound of $h$ \\
\hline [21] & 1.5 \\
Theorem 3, $m=1$ & 2 \\
Theorem 3, $m=4$ & 3.16 \\
Theorem 3, $m=5$ & 8.8 \\
\hline
\end{tabular}

Rule 2: IF $x_{2}(t)$ is $M_{2}$, THEN

$$
\begin{gathered}
\dot{x}(t)=A_{2} x(t)+A_{d 2} x(t-h)+B_{2} u(t)+B_{12} \omega(t), \\
y(t)=C_{2} x(t)+D_{2} \omega(t)
\end{gathered}
$$

with

$$
\begin{array}{cc}
A_{1}=\left[\begin{array}{cc}
-0.1125 & -0.02 \\
1 & 0
\end{array}\right], \quad A_{2}=\left[\begin{array}{cc}
-0.1125 & -1.527 \\
1 & 0
\end{array}\right], \\
A_{d 1}=\left[\begin{array}{cc}
-0.0125 & -0.005 \\
0 & 0
\end{array}\right], & A_{d 2}=\left[\begin{array}{cc}
-0.0125 & -0.23 \\
0 & 0
\end{array}\right], \\
B_{1}=B_{2}=\left[\begin{array}{l}
1 \\
1
\end{array}\right], & B_{11}=B_{12}=I_{2}, \\
C_{1}=C_{2}=D_{1}=D_{2}=I_{2} .
\end{array}
$$

The membership function is

$$
M_{11}\left(x_{2}(t)\right)=1-\frac{x_{2}^{2}(t)}{2.25}, \quad M_{21}\left(x_{2}(t)\right)=\frac{x_{2}^{2}(t)}{2.25} \text {. }
$$

The initial state is

$$
x(0)=\left[\begin{array}{ll}
-1 & -1.2
\end{array}\right]^{T}, \quad \omega(t)=\left[\begin{array}{ll}
\frac{\sin (3 t)}{\exp (t)} & \frac{\cos (3 t)}{\exp (t)}
\end{array}\right]^{T} .
$$

First, we should find the allowable maximum time delay $h$ which let the fuzzy system passive. Table 1 shows the maximum time delay obtained by [21] and our method.

It clearly shows that the method in our paper can get larger upper bound than before. It also shows that conservatism is further reduced when $m$ increases.

Then we let $h=1.5, m=4$, and we can obtain

$$
P=\left[\begin{array}{ll}
9.4980 & 9.1547 \\
9.1547 & 8.8849
\end{array}\right], \quad X=\left[\begin{array}{ll}
0.6351 & 0.4997 \\
0.5807 & 0.4826
\end{array}\right] .
$$

The fuzzy controller gains by our method are given by

$$
\begin{aligned}
& K 1=\left[\begin{array}{ll}
150.7358 & -218.3595
\end{array}\right], \\
& K 2=\left[\begin{array}{ll}
150.9502 & -219.0519
\end{array}\right] .
\end{aligned}
$$

Figure 3 shows the state response $x_{1}(t)$ of the closed-loop system with the controller gains in (58), and Figure 4 shows the state response $x_{2}(t)$ of the closed-loop system. 


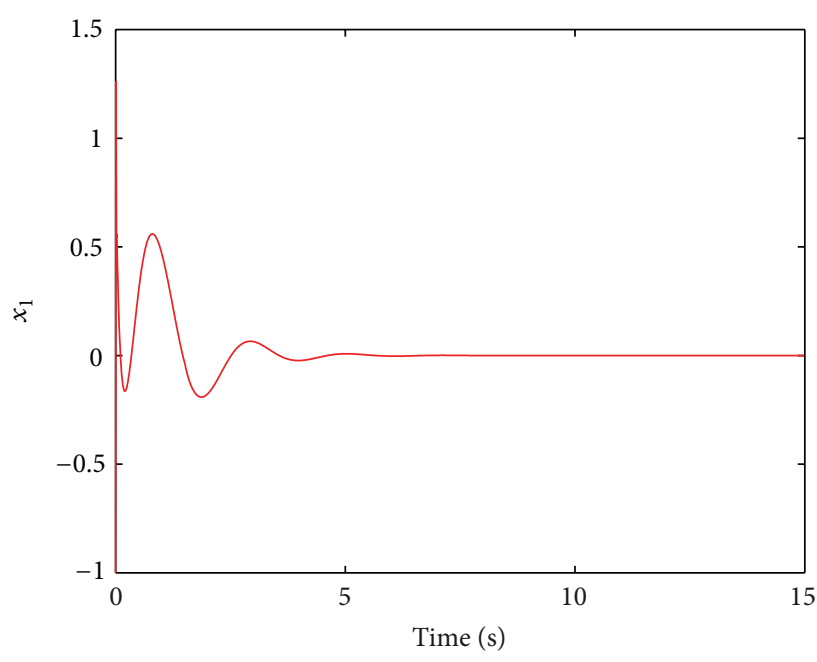

FIGURE 3: State response $\times 1$ of the system.

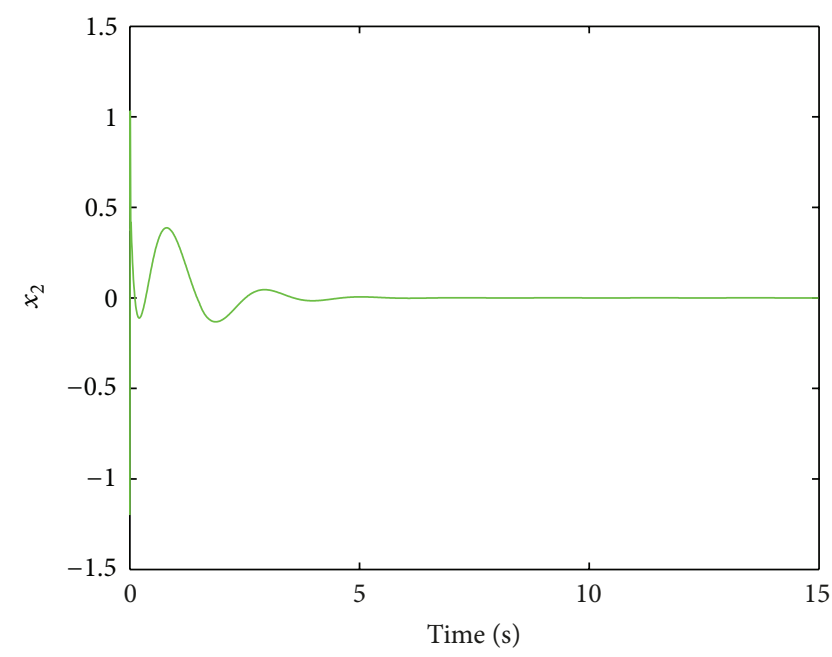

FIGURE 4: State response $\times 2$ of the system.

\section{Conclusion}

This paper has adopted the delay partitioning approach to analyse the passivity and passification of delay fuzzy system based on T-S model. The theorems given in this paper are all in terms of LMIs. Examples have illustrated the effectiveness of our results. The method in our paper has further reduced the conservatism and the effect has been more apparent when $m$ increases. In addition, the results obtained in this paper also can be extended to the fuzzy system with time-varying delay and uncertainties.

\section{Acknowledgments}

This work was supported in part by the National Natural Science Foundation of China under Grant 61273144 and 61203041, the Natural Science Foundation of Beijing under Grant 4122071, the Chinese National Post-doctor Science Foundation under Grants 2011M500217 and 2012T50036, and the Doctoral Fund of Ministry of Education of China under Grant 20120036120013.

\section{References}

[1] J. C. Willems, "Dissipative dynamical systems. I. General theory," Archive for Rational Mechanics and Analysis, vol. 45, pp. 321-351, 1972.

[2] D. J. Hill and P. J. Moylan, "Dissipative dynamical systems: basic input-output and state properties," Journal of the Franklin Institute, vol. 309, no. 5, pp. 327-357, 1980.

[3] G. Feng, "A survey on analysis and design of model-based fuzzy control systems," IEEE Transactions on Fuzzy Systems, vol. 14, no. 5, pp. 676-697, 2006.

[4] J. Lam and S. Zhou, "Dynamic output feedback $H_{\infty}$ control of discrete-time fuzzy systems: a fuzzy-basis-dependent Lyapunov function approach," International Journal of Systems Science, vol. 38 , no. 1, pp. 25-37, 2007.

[5] Y. He, M. Wu, J.-H. She, and G.-P. Liu, "Parameter-dependent Lyapunov functional for stability of time-delay systems with polytopic-type uncertainties," Institute of Electrical and Electronics Engineers, vol. 49, no. 5, pp. 828-832, 2004.

[6] E. Kim and H. Lee, "New approaches to relaxed quadratic stability condition of fuzzy control systems," IEEE Transactions on Fuzzy Systems, vol. 8, no. 5, pp. 523-534, 2000.

[7] X. N. Song, J. W. Lu, S. Y. Xu, H. Shen, and J. J. Lu, "Robust stabilization of state delayed T-S fuzzy systems with input saturation via dynamic anti-windup fuzzy design," International Journal of Innovative Computing, Information and Control, vol. 7, no. 12, pp. 6665-6676, 2011.

[8] Y. Y. Cao and P. M. Frank, "Robust $H_{\infty}$ disturbance attenuation for a class of uncertain discrete-time fuzzy systems," IEEE Transactions on Fuzzy Systems, vol. 8, no. 4, pp. 406-415, 2000.

[9] C. Lin, Q.-G. Wang, and T. H. Lee, "Improvement on observerbased $H_{\infty}$ control for T-S fuzzy systems," Automatica, vol. 41, no. 9, pp. 1651-1656, 2005.

[10] P. Shi and S. K. Nguang, " $H_{\infty}$ output feedback control of fuzzy system models under sampled measurements," Computers \& Mathematics with Applications, vol. 46, no. 5-6, pp. 705-717, 2003.

[11] S. K. Nguang, P. Shi, and S. Ding, "Delay-dependent fault estimation for uncertain time-delay nonlinear systems: an LMI approach," International Journal of Robust and Nonlinear Control, vol. 16, no. 18, pp. 913-933, 2006.

[12] D. F. Zhang, H. Wang, B. C. Lu, and Z. Q. Wang, "LMI-based fault detection fuzzy observer design with multiple performance constraints for a class of non-linear systems: Comparative Study," International Journal of Innovative Computing, Information and Control B, vol. 8, no. 1, pp. 633-645, 2012.

[13] X. M. Zhang, Z. J. Zhang, and G. P. Lu, "Fault detection for statedelay fuzzy systems subject to random communication delay," International Journal of Innovative Computing, Information and Control, vol. 8, no. 4, pp. 2439-2451, 2012.

[14] X. J. Su, L. G. Wu, P. Shi, and Y. D. Song, " $H_{\infty}$ model reduction of Takagi-Sugeno Fuzzy Stochastic Systems," IEEE Transactions on Systems, Man, and Cybernetics B, vol. 42, no. 6, pp. 1574-1585, 2012.

[15] K. Tanaka and H. Wang, Fuzzy Control Systems Design and Analysis: A Linear Matrix Inequality Approach, New York, NY, USA, 2001. 
[16] S. Xu, J. Lam, S. Huang, and C. Yang, " $H_{\infty}$ model reduction for linear time-delay systems: continuous-time case," International Journal of Control, vol. 74, no. 11, pp. 1062-1074, 2001.

[17] Y. He, Q. G. Wang, and C. Lin, "An improved Ho filter design for systems with time-varying interval delay," IEEE Transactions on Circuits and Systems II, vol. 53, no. 11, pp. 1235-1239, 2006.

[18] X. J. Su, P. Shi, L. G. Wu, and Y. D. Song, "A novel approach to filter design for T-S fuzzy discrete-time systems with timevarying delay," IEEE Transactions on Fuzzy Systems, vol. 20, no. 6, pp. 1114-1129, 2012.

[19] C. Li and X. Liao, "Passivity analysis of neural networks with time delay," IEEE Transactions on Circuits and Systems II, vol. 52, no. 8, pp. 471-475, 2005.

[20] C. Li, H. Zhang, and X. Liao, "Passivity and passification of fuzzy systems with time delays," Computers \& Mathematics with Applications, vol. 52, no. 6-7, pp. 1067-1078, 2006.

[21] Y. Zhang, Q. L. Zhang, and Q. Li, "Passive controller design for T-S fuzzy systems with time delay," Acta Automatica Sinica, vol. 35, no. 3, pp. 328-331, 2009.

[22] C. Li, H. Zhang, and X. Liao, "Passivity and passification of uncertain fuzzy systems," IEE Proceedings Circuits, Decices and Systems, vol. 152, no. 6, pp. 649-653, 2005. 


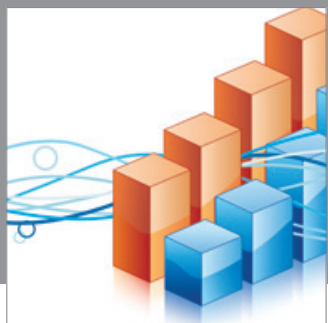

Advances in

Operations Research

mansans

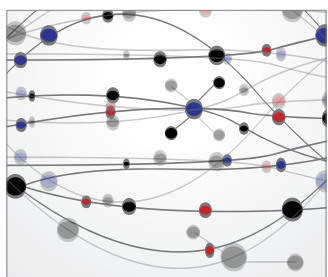

The Scientific World Journal
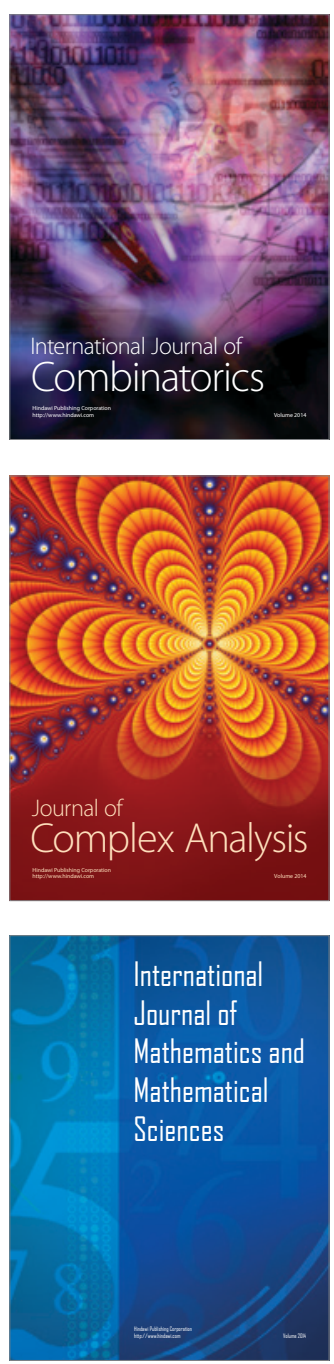
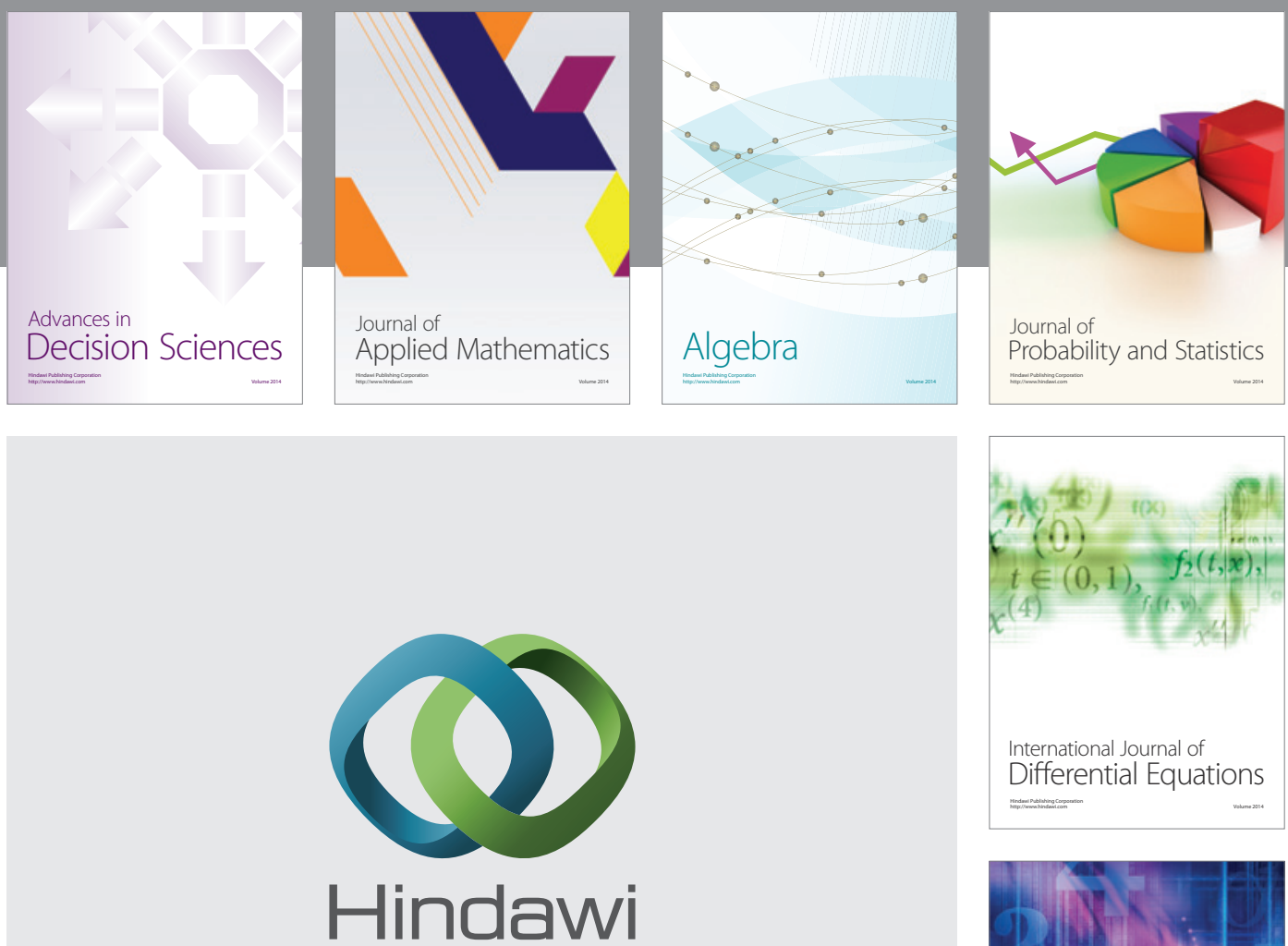

Submit your manuscripts at http://www.hindawi.com
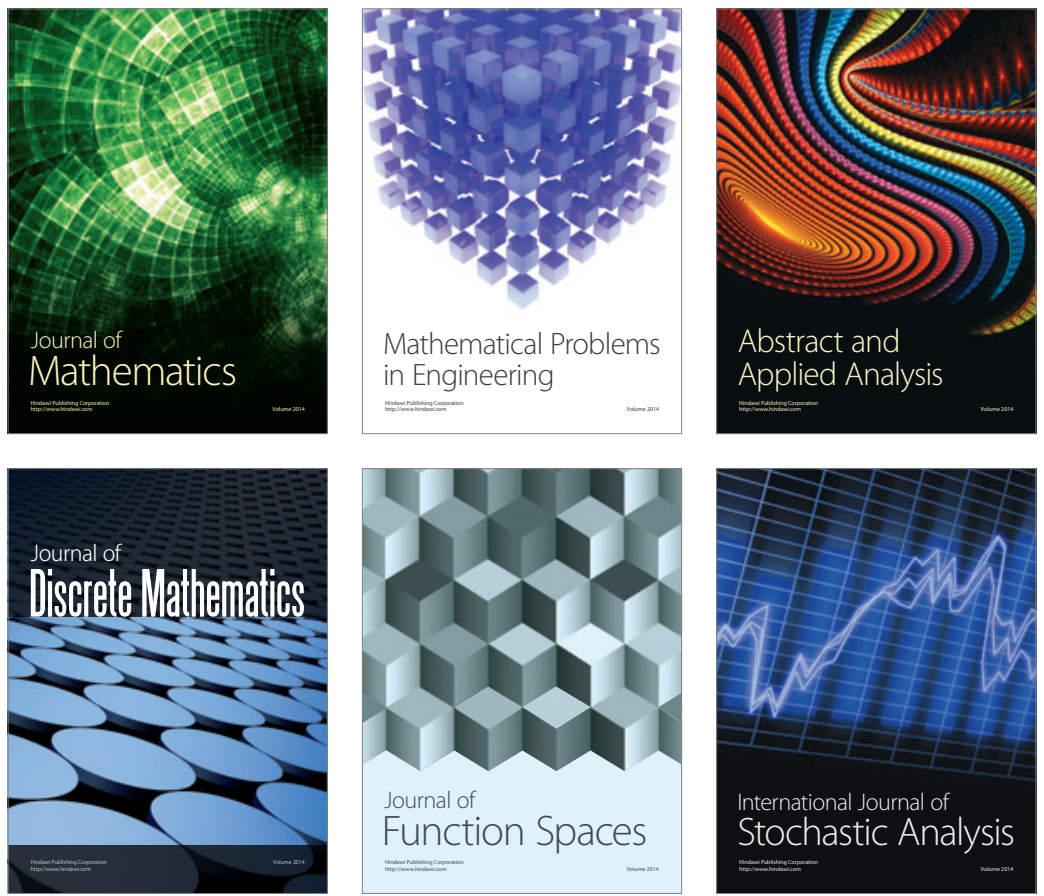

Journal of

Function Spaces

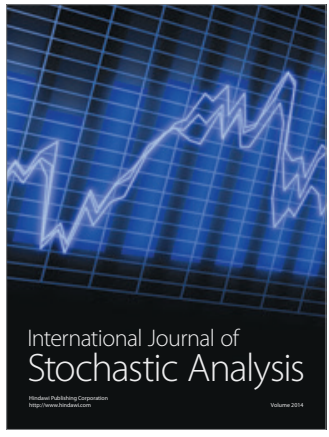

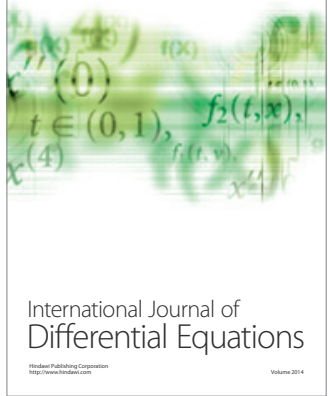
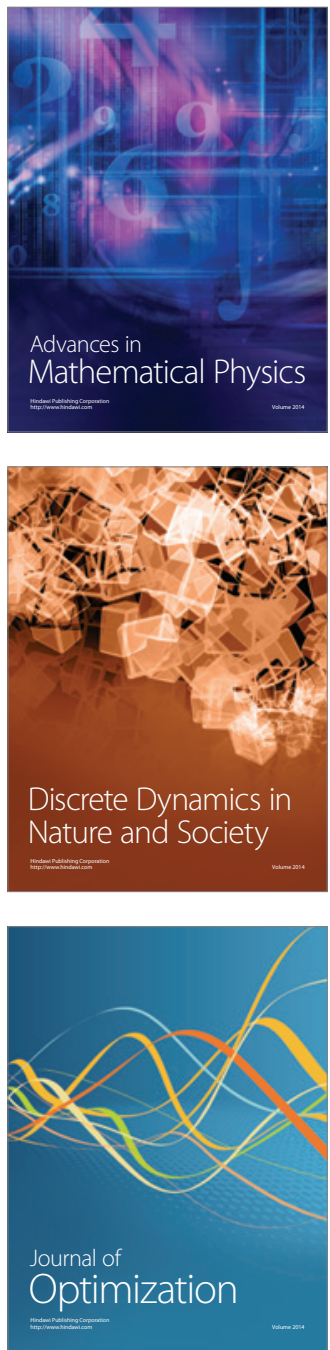